\section{Another X-ray Galaxy ?}

THE detection of $\mathrm{X}$-rays from the radio galaxy Centaurus A by C. S. Bowyer's group at the University of California, Berkeley, brings to three the number of extragalactic objects with associated X-ray emission. There are, of course, thirty odd X-ray sources known to be probably within the Milky Way galaxy, of which the strongest appears to be Scorpius X-1. But Centaurus $\mathrm{A}$ at a distance of twelve million light years seems to be intrinsically of the order of $10^{5}$ times more powerful than Sco X-1. With the result of Bowyer, M. L. Lampton and J. E. Mack indicating that the $\mathrm{X}$-ray emission from the radio galaxy Centaurus A is approximately twice the radio emission, it seems that Centaurus $\mathrm{A}$, one of the brightest objects in the radio sky, ought more properly to be called an X-ray galaxy.

Its peculiar appearance at optical wavelengths as much as anything has been the stimulus to observe Centaurus A with X-ray equipment, but so far the results have been inconclusive. The California telescope flown on an Aerobee rocket from Natal, Brazil, last summer measured a flux of 0.04 photons $\mathrm{cm}^{-2} \mathrm{~s}^{-1}$ making the X-ray emission from Centaurus A roughly a third of that from the X-ray galaxy M87. The third extragalactic $\mathrm{X}$-ray object is the quasar $3 \mathrm{C} 273$, and all three were detected during the flight. Clearly the sensitivity of the California telescope coupled to the technique of concentrating on just three sources rather than allowing the telescope to

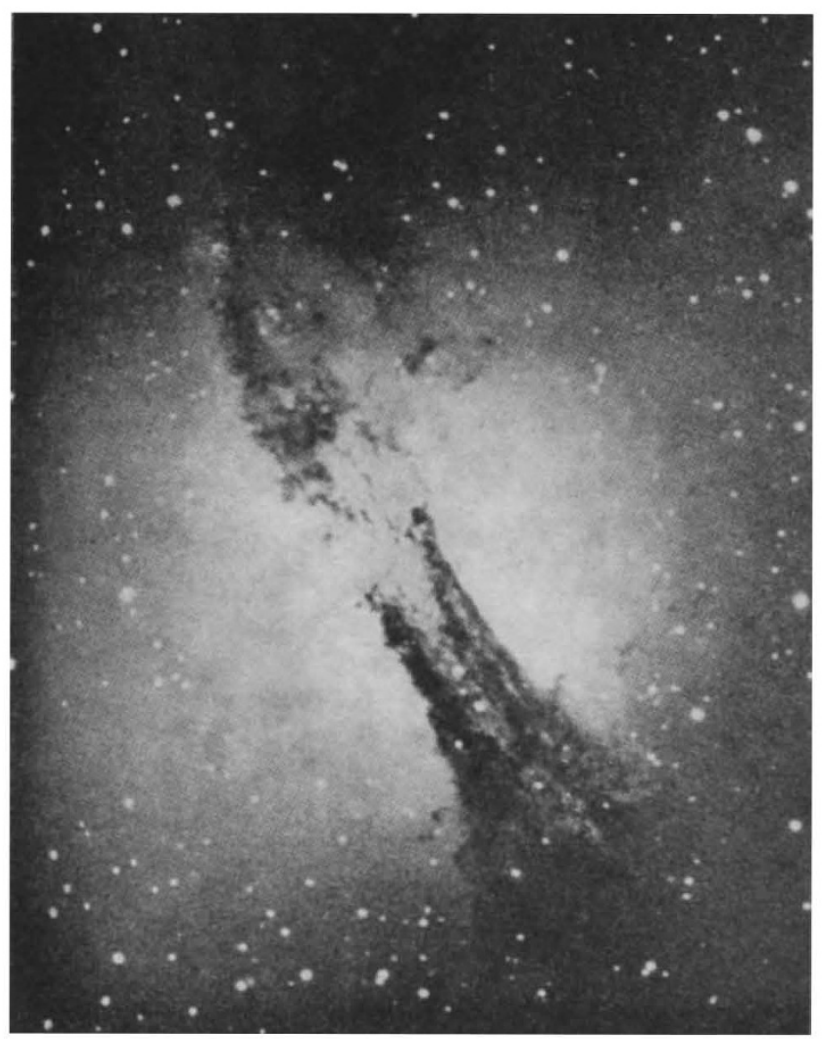

Centaurus A: An X-ray galaxy? scan across the sky tipped the balance in favour of tho detection of Centaurus A.

The optical characteristic of Centaurus A is the dark lane running across the galaxy, but at radio wavelengths the pattern is the familiar one of a double radio source about three million light years across with the optical galaxy at the centre. In the case of Centaurus A, however, the optical galaxy marks the midpoint of a second double radio source which is much nearer the optical object. From the current dogma of exploding galaxies the inference is that Centaurus A must have exploded twice.

Another stimulus behind the search for extragalactic $\mathrm{X}$-ray objects is to see whether sources outside the Milky Way can account for the isotropic X-ray background. 'This would require galaxies to emit something like $10^{2}$ to $10^{3}$ more energy in $\mathrm{X}$-rays than at longer wavelengths. But with the ratio being more like fifty for M87 and about unity for Centaurus A this hypothesis now looks less probable than the alternative based on Compton interactions between cosmic ray electrons and the microwave background.

\section{SINGULARITIES \\ How to Spof a Black Hole}

\section{from our Cosmology Correspondent}

INTEREST in the existence of "black holes" of collapsed matter, which was recently stimulated by LyndenBell's suggestion that such holes could be associated with quasars and galaxy centres, will receive a further boost as a result of the relative ease of some observational tests which have now been proposed by A. A. Wyller (Astrophys. J., 160, 443; 1970). Wyller is particularly concerned with the possible existence of black holes in globular clusters, where they may be responsible for the brightness excesses observed at the cluster centres. Postulating that a supermassive object has collapsed into a singularity in such a cluster, Wyller examines in detail spectroscopic and gravitational tests for such an object; these are marginally possible with present-day techniques, so that there is every chance of a positive result being achieved as future developments make observations easier.

The holes considered have masses comparable to a few per cent of average globular cluster masses, which are themselves $\sim 10^{5} \mathrm{M}_{\odot}$, so that their radii are in the range $0.01-1 \cdot 0 \mathrm{R}_{0}$. For the particular case of 47 Tuc, where the spectroscopic possibilities are best, as a result of the cluster's large size and proximity to the solar system, an analysing diaphragm of $2^{\prime \prime}$ would give a sampling radius of $10^{6} R_{\odot}$ about the hypothetical black hole, in which range it should be possible to detect the contribution of orbital Doppler shifts in the spectra of the stars close to the central singularity in the line spectra. Measurement of a gravitational redshift would be more difficult, but this might prove detectable as a steady component while the Doppler component varics cyclically over a few years. Wyllers' calcula- 